\title{
Senile Systemic Amyloidosis in an Aged Savannah Monkey (Cercopithecus aethiops) with Tenosynovial Degeneration
}

\author{
James Kenn CHAMBERS ${ }^{1}$, Takuya KANDA ${ }^{1)}$, Atsushi SHIRAI ${ }^{2}$, Keiichi HIGUCHI $^{3)}$, Shu-ichi IKEDA $^{4)}$ and \\ Yumi UNE ${ }^{1)}$ \\ ${ }^{1)}$ Laboratory of Veterinary Pathology, School of Veterinary Medicine, Azabu University, 1-17-71 Fuchinobe, Sagamihara, Kanagawa \\ 229-8501, ${ }^{2)}$ Omiya Park Zoo, 4 Takahanacho, Omiyaku, Saitama 330-0803, ${ }^{3)}$ Department of Aging Biology, Institute on Aging and \\ Adaptation, Shinshu University Graduate School of Medicine, 3-1-1 Asahi, Matsumoto, Nagano 390-8621 and ${ }^{4)}$ Department of \\ Medicine, Shinshu University School of Medicine, 3-1-1 Asahi, Matsumoto Nagano 390-8621, Japan
}

(Received 4 September 2009/Accepted 22 December 2009/Published online in J-STAGE 15 January 2010)

ABSTRACT. Senile systemic amyloidosis (SSA) is a rather common disease in elderly people, but it is very rare in animals, including nonhuman primates. Pathological examination of a 26-year-old male savannah monkey (Cercopithecus aethiops) revealed systemic amyloidosis with severe cardiac fibrosis, and tenosynovial degeneration of the elbow and knee joints. The amyloid deposits were observed predominantly in the heart, lung, intestine and tenosynovium, and were positive for transthyretin (TTR) in immunohistochemistry. Immunohistochemical results, together with the distribution of the amyloid deposited lesions and the age of the monkey, were equivalent to those of human SSA. This is the second case of animal SSA with unprecedented TTR amyloid deposited lesions of the tenosynovium resembling human SSA. There may be a genetic factor that makes this species susceptible to SSA, since SSA has been reported in no other mammal besides humans.

KEY WORDS: Cercopithecus aethiops, senile systemic amyloidosis, tenosynovial amyloidosis, transthyretin.

J. Vet. Med. Sci. 72(5): 657-659, 2010

Senile Systemic Amyloidosis (SSA) is an intractable disease that afflicts elderly people [16]. Amyloid derived from wild type transthyretin (TTR) is deposited predominantly in the heart, and therefore this disorder is also referred to as Senile Cardiac Amyloidosis (SCA) [15]. In severe cases, the prognosis is quite poor due to cardiac dysfunction [16]. TTR-related amyloid is also deposited in other organs such as the lungs, intestinal tract, and joint capsule and tenosynovium, although the kidneys and the liver are less likely to be involved $[2,3,6,13]$. Some reports indicate that $25 \%$ of people over the age of 80 are affected with SSA to some extent, and its prevalence is related to particular genetic variations $[2,15]$. However, SSA in animals has not been reported except for one vervet monkey (Cercopithecus aethiops), and the question why the human is the only species that is commonly affected by this disease still remains [8]. To our knowledge, any kind of systemic amyloidosis has not been reported in Cercopithecus aethiops except for the previous case report of SSA. Here we report the second case of SSA in Cercopithecus aethiops with unprecedented TTR amyloid deposition in the tenosynovium.

A 26-year-old male savannah monkey (Cercopithecus aethiops), in a zoo in Japan, gradually lost its physical strength over a two-month period. The monkey died after suffering two weeks of coughing, shortness of breath, and watery nasal discharge. Necropsy was performed on the day after death. Major organs were collected, fixed in 10\% neutral buffered formalin, and conventional paraffin embedded

\footnotetext{
* Correspondence to: Une, Y., Laboratory of Veterinary Pathology, School of Veterinary Medicine, Azabu University, 1-17-71 Fuchinobe, Sagamihara, Kanagawa 229-8501, Japan. e-mail: une@azabu-u.ac.jp
}

sections were made. Hematoxylin and eosin (HE) staining and some other additional staining was performed, including Congo red, Azan and immunohistochemical staining. In the immunoperoxidase technique, sections were treated with formic acid for antigen retrieval and subsequently incubated with primary antibodies at $4^{\circ} \mathrm{C}$ overnight. Antibodies against TTR (provided by Dr. Hoshii of Yamaguchi University) and Amyloid A (AA) protein (Kyowa Medex Co., Tokyo) were used as primary antibodies at dilution of $1: 4000$ and 1:300 respectively. A Histofine simple stain MAX-PO kit (Nichirei, Tokyo, Japan) and 3'3-diaminobenzidine were used to visualize the immunohistochemical reaction.

The monkey weighed $3.7 \mathrm{~kg}$ at necropsy with slight emaciation. The face was smeared with blood, from the nostrils to the mandible, demonstrating severe hemoptysis. Clear yellow pleural fluid filled the entire thoracic cavity (left thoracic cavity: $156 \mathrm{ml}$; the right cavity was not determined). Both the atria and ventricles were severely dilated, allowing external recognition of the intraventicular blood clot (Fig. 1A). The lungs were highly edematous and hemorrhagic on the cut surface. Hemorrhagic lesions were also observed in the synovium and tendons of both elbows and knees (Fig. 1B).

Histological examination revealed severe fibrosis throughout the ventricular wall (Fig. 1C). Diffuse congophilic deposits were observed in small arteries and arterioles, in the myocardial interstitium, and in the cardiac valves (Figs. 1D, 2). Some arteries were constricted by patchy depositions in the arterial wall. Remaining cardiomyocytes were hypertrophic with bizarrely shaped nuclei and pale cytoplasm. Laminar deposits of Congophilic amyloid were seen in the collagen fibers of the tendons of both knee and elbow joints (Fig. 1E), accompanied by rupture of 

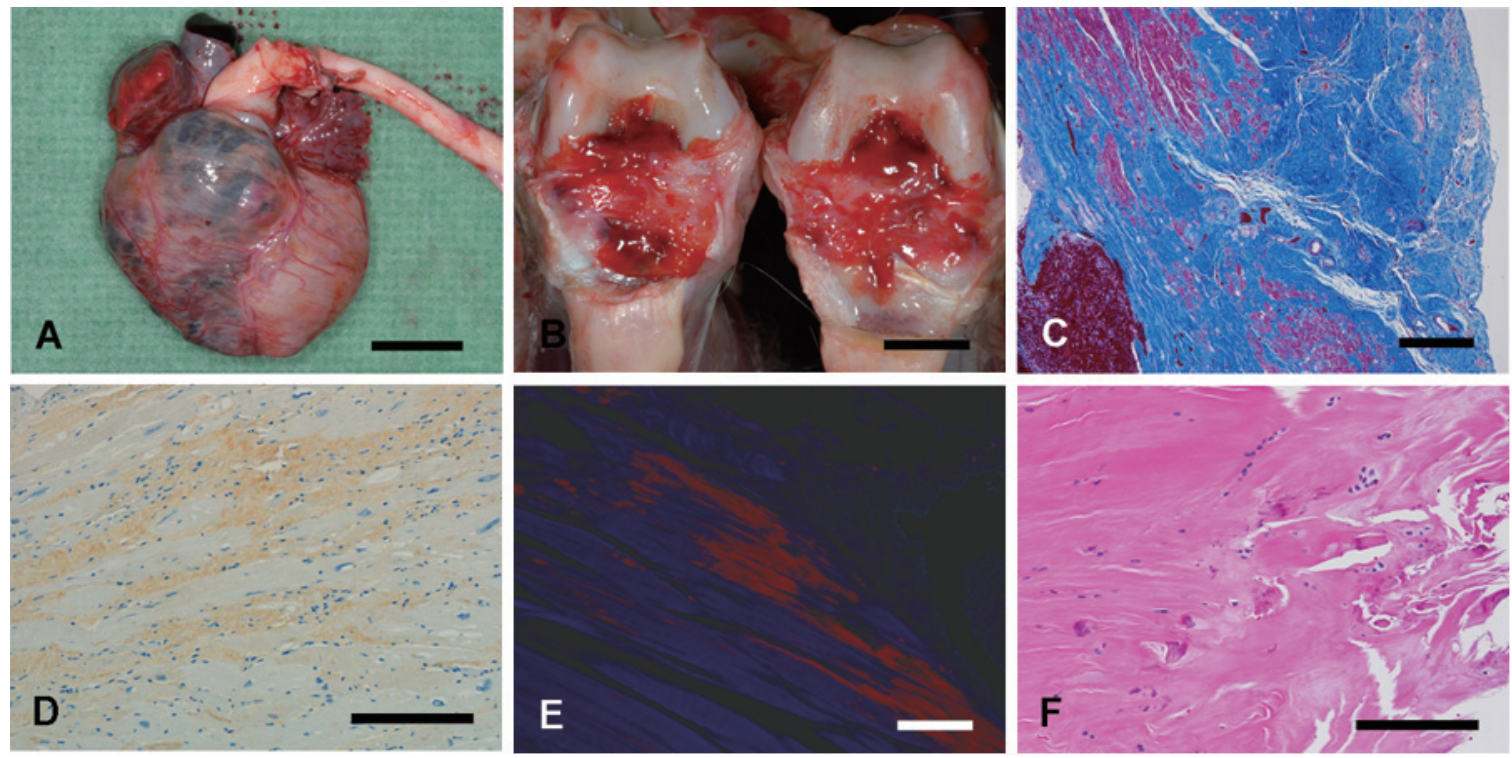

Fig. 1. (A) Both the atria and ventricles were severly dilated, allowing external recognition of the intraventicular blood clot. Bar=20 mm. (B) Hemorrhage in the synovium of the knee. Bar=10 mm. (C) Fibrosis replaced the entire left ventricular wall. Azan stain, bar $=500 \mu \mathrm{m}$. (D) Congophilic deposits in the myocardial interstitium were positive for TTR when examined immunohistochemically. Bar $=100 \mu \mathrm{m}$. (E) Laminar amyloid deposition in the collagen fibers. Tendon of the knee joint. Fluorescence microscope, Congo red, bar $=250 \mu \mathrm{m}$. (F) Rupture of the collagen fibers with consequent microcalcification. Tendon of the knee joint. HE, bar $=100 \mu \mathrm{m}$.

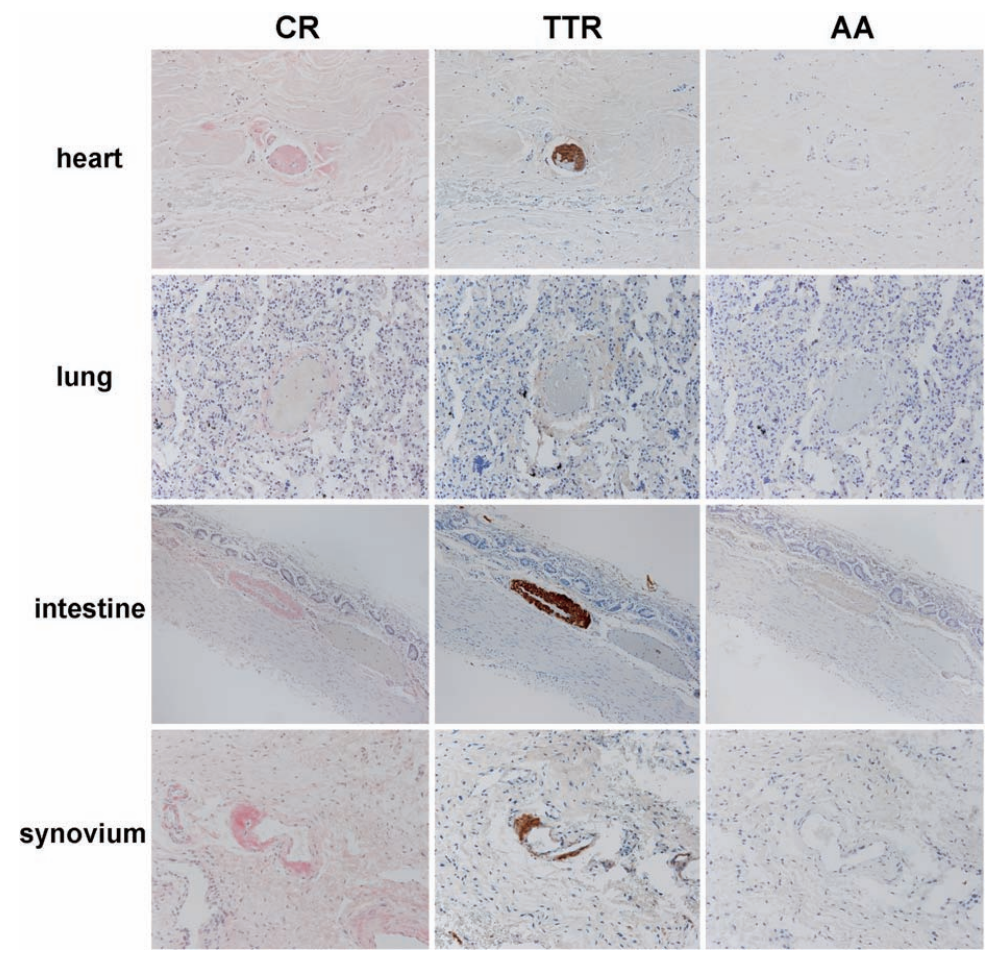

Fig. 2. Congophilic amyloid deposits of the heart, lungs, intestine, and synovium were positive for TTR and negative for AA when examined immunohistochemically. 
the tendons with consequent microcalcification and cartilaginous metaplasia (Fig. 1F). Amyloid deposition in the vascular walls was also seen in the synovium of both elbow and knee joints, lungs and gastrointestinal tract to a lesser degree (Fig. 2). In contrast, hepatic, splenic, and renal involvement was scarce. Congophilic amyloid deposits in the heart, lungs, intestines and synovium were positive for TTR and negative for AA when examined immunohistochemically (Fig. 1D, 2). However, some of the congophilic deposits did not react to either of the antibodies.

Most cases of systemic amyloidosis in animals, including monkeys, are AA amyloidosis [1, 5]. However, the liver, spleen, and kidneys, all at which are the main organs afflicted in AA amyloidosis, were not involved in our case. Immunohistochemical results, together with the distribution of the lesions and the age of the monkey, were equivalent to those of human SSA. Congestive heart failure accompanied by arrythmia and myocardial infarcts are of prognostic significance in human SSA patients [4, 7]. This case represents an end stage heart failure, resembling its human counterpart.

SSA is currently an incurable aging disease awaiting further study [10]. As mentioned above, this is the second report of SSA in animals, occurring as before in Cercopithecus aethiops, with unprecedented TTR-derived amyloid deposition in the tenosynovium, which is one of the locations affected in humans $[3,6]$. Both of these two monkeys were male, the same gender that has the higher risk for myocardial involvement of SSA in humans [9]. As it was seen in this case, amyloids are deposited in the vessels and ligaments of the joints also in humans [11]. Our findings indicate that the monkey died from pulmonary hemorrhage and hydrothorax, as a consequence of severe congestive heart failure and amyloid degeneration of the arterioles in the lungs. SSA in human is well known to affect the heart and, to a lesser degree, the lungs, intestine, and synovium. Especially in humans, wild type TTR-related amyloid deposition on tenosynovium is recently surmised to be an initial manifestation of SSA $[12,13]$. We considered the tenosynovial rupture and degeneration of the monkey knees and elbows as a consequence of deterioration of the ligament due to substantial amyloid burden. TTR-derived amyloid deposition in the carpal joint ligament causes dysesthesia in humans $[12,13]$, however, we did not find any neurologic manifestations clinically or pathologically in this case.

Cercopithecus aethiops is the only species reported to develop SSA essentially identical to that of humans. Molecular biological investigation of TTR in this species may have implications for the etiology of this disease in both animals and humans.

ACKNOWLEDGMENT. The authors thank Dr. Yoshinobu Hoshii of Yamaguchi University for providing the valuable antibody for TTR.

\section{REFERENCES}

1. Blanchard, J. L., Baskin, G. B. and Watson, E. A. 1986. Gen- eralized amyloidosis in rhesus monkeys. Vet. Pathol. 23: 425430.

2. Cornwell, G. G3rd., Murdoch, W. L., Kyle, R. A., Westermark, P. and Pitkanen, P. 1983. Frequency and distribution of senile cardiovascular amyloid. A clinicopathologic correlation. Am. J. Med. 75: 618-623.

3. Goffin, Y. A., McCrickard, E. L., Ameryckx, J. P., Malmendier, C. L., Hiden, M. and Cornwell, G. G3rd. 1985. Amyloidosis of the joints: evidence that human hip capsules have a unique predisposition for amyloid of the senile systemic type. Appl. Pathol. 3: 88-95.

4. Johansson, B. and Westermark, P. 1991. Senile systemic amyloidosis: A clinico-pathological study of twelve patients with massive amyloid infiltration. Int. J. Cardiol. 32: 83-92.

5. Johnson, K. H., Westermark, P., Sletten, K. and O'brien, T. D. 1996. Amyloid proteins and amyloidosis in domestic animals. Amyloid. 3: 270-289.

6. Kyle, R. A., Gertz, M. A. and Linke, R. P. 1992. Amyloid localized to tenosynovium at carpal tunnel release. Immunohistochemical identification of amyloid type. Am. J. Clin. Pathol. 97: $250-253$

7. Kyle, R. A., Spittell, P. C., Gertz, M. A., Li, C. Y., Edwards, W. D., Olson, L. J. and Thibodeau, S. N. 1996. The premortem recognition of systemic senile amyloidosis with cardiac involvement. Am. J. Med. 101: 395-400.

8. Nakamura, S., Okabayashi, S., Ageyama, N., Koie, H., Sankai, T., Ono, F., Fujimoto, K. and Terao, K. 2008. Transthyretin amyloidosis and two other aging-related amyloidoses in an aged vervet monkey. Vet. Pathol. 45: 67-72.

9. Rapezzi, C., Riva, L., Quarta, C. C., Perugini, E., Salvi, F., Longhi, S., Ciliberti, P., Pastorelli, F., Biagini, E., Leone, O., Cooke, R. M., Bacchi-Reggiani, L., Ferlini, A., Cavo, M., Merlini, G., Perlini, S., Pasquali, S. and Branzi, A. 2008. Genderrelated risk of myocardial involvement in systemic amyloidosis. Amyloid. 15: 40-48.

10. Sekijima, Y., Kelly, J. W. and Ikeda, S. 2008. Pathogenesis of and therapeutic strategies to ameliorate the transthyretin amyloidoses. Curr. Pharm. Des. 14: 3219-3230.

11. Stein, K., Storkel, S., Linke, R. P. and Goebel, H. H. 1987. Chemical heterogeneity of amyloid in the carpal tunnel syndrome. Virchows Arch. A Pathol. Anat. Histopathol. 412: 3745 .

12. Takei, Y., Hattori, T., Goto, T., Tokuda, T., Saitoh, S., Hoshii, Y. and Ikeda, S. 2002. Senile systemic amyloidosis presenting as bilateral carpal tunnel syndrome. Amyloid. 9: 252-255.

13. Takei, Y., Hattori, T., Tokuda, T., Matsuda, M., Saitoh, S. Hoshii, Y. and Ikeda, S. 2003. Senile systemic amyloidosis presenting as bilateral carpal tunnel and left ulnar tunnel syndrome. Intern. Med. 42: 1050-1051.

14. Tanskanen, M., Peuralinna, T., Polvikoski, T., Notkola, IL., Sulkava, R., Hardy, J., Singleton, A., Kiuru-Enari, S., Paetau, A., Tienari, P. J. and Myllykangas, L. 2008. Senile systemic amyloidosis affects $25 \%$ of the very aged and associates with genetic variation in alpha2-macroglobulin and tau: a population-based autopsy study. Ann. Med. 40: 232-239.

15. Westermark, P., Sletten, K., Johansson, B. and Cornwell, G. G3rd. 1990. Fibril in senile systemic amyloidosis is derived from normal transthyretin. Proc. Natl. Acad. Sci. U.S.A. 87: 2843-2845.

16. Westermark, P., Bergstrom, J., Solomon, A., Murphy, C. and Sletten, K. 2003. Transthyretin-derived senile systemic amyloidosis: clinicopathologic and structural considerations. Amyloid. 10: (Suppl. 1) 48-54. 